\title{
Capsule Commentary on Katzman et al., Army and Navy ECHO Pain Telementoring Improves Clinician Opioid Prescribing for Military Patients: An Observational Cohort Study
}

\author{
Bixuan Lin, MD \\ $\mathrm{J}$ Gen Intern Med 34(3):445 \\ DOI: $10.1007 / \mathrm{s} 11606-018-4762-6$ \\ () Society of General Internal Medicine 2019
}

Medical College of Wisconsin and Affiliated Hospitals, Milwaukee, USA.

$\mathrm{T}$ his study by Katzman et al. ${ }^{1}$ examined the effects of the ECHO Pain Telementoring program on providers' habits in prescribing opioids. Providers voluntarily attended 2-h educational sessions focused on pain management and opioid use, and changes in opioid prescription behavior were measured and compared to those of providers who did not participate. Providers who received ECHO Pain training had significantly greater decreases in opioid prescribing compared to the control group, including average number of prescriptions/patient/year, reduction in morphine milligrams equivalent (MME)/patient/year, and concurrent benzodiazepine prescription.

This investigation supports the benefit of regimented pain management education on decreasing opioid prescriptions. However, participants could be different than nonparticipants in a number of ways that would affect the study outcomes, so one should be cautious about applying these results. A randomized, mandatory educational intervention could have provided more definite evidence for benefit. In addition, further studies could better elucidate how prescriptions were reduced - whether the decreases happened independently, or if other interventions shown to decrease opioid needs, such as physical therapy ${ }^{2}$ or psychosocial support ${ }^{3}$ occurred. Provider feedback regarding which aspects of the sessions were impactful could help identify high-yield teaching points and improve training efficiency. In addition, comparison of the change in prescribing patterns among providers attending varying number of classes may show whether there is increased benefit of attending more sessions or whether there is a point of diminishing return.

Moreover, clinical outcomes that result from the decreased opioids prescribed should be further studied. For instance, in both provider groups, average MME was well over $50 \mathrm{mg}$, the defined safe daily dose. ${ }^{4}$ Comparing the decrease among patients exceeding $50 \mathrm{mg}$ daily MME could be useful. In addition, the ultimate study would explore the relationship between participation in ECHO Pain programs and drug overdose rates.

ECHO Pain Telementoring may reduce opioid prescribing. A deeper look into how providers' pain management strategies were changed by the sessions, as well as how clinical outcomes are affected, would lead to better understanding of why the program is effective and how it could potentially be improved.

Corresponding Author: Bixuan Lin, MD; Medical College of Wisconsin and Affiliated Hospitals, Milwaukee, USA (e-mail: bilin@mcw.edu).

\section{Compliance with Ethical Standards:}

Conflict of Interest: The author declares that she does not have a conflict of interest.

Publisher's Note Springer Nature remains neutral with regard to jurisdictional claims in published maps and institutional affiliations.

\section{REFERENCES}

1. Katzman JG, Gualls CR, Shatterfield CR, Kistin M, Hofmann K, Greenberg N, Swift R, Comerci GD, Fowler R, Arora S. Army and Navy ECHO Pain Telementoring Improves Clinician Opioid Prescribing for Military Patients: An Observational Cohort Study. J Gen Intern Med. doi:https://doi.org/10.1007/s11606-018-4710-5

2. Brose SW, Schneck H, Bourbeau CJ. An Interdisciplinary Approach to Reducing Opioid Prescriptions to Patients with Chronic Pain in a Spinal Cord Injury Center. PM R. 2018;S1934-1482(18):30930-30934. doi:https://doi.org/10.1016/j.pmrj.2018.09.030

3. Launonen E, Wallace I, Kotovirta E, Alho H, Simojoki K. Factors associated with non-adherence and misuse of opioid maintenance treatment medications and intoxicating drugs among Finnish maintenance treatment patients. Drug Alcohol Depend. 2016;162:227-235. doi:https:// doi.org/10.1016/j.drugalcdep.2016.03.017

4. Adewumi AD, Hollinworth SA, Maravilla JC, Connor JP, Atali R. Prescribed Dose of Opioids and Overdose: A Systematic Review and Meta-Analysis of Unintentional Prescription Opioid Overdose. CNS Drugs. 2018;32(2):101-116. doi:https://doi.org/10.1007/s40263-018-0499-3 